\title{
Necrophagous species of Diptera and Coleoptera in northeastern Brazil: state of the art and challenges for the Forensic Entomologist
}

\author{
Simão D. Vasconcelos ${ }^{1,2} \&$ Manuela C. S. Araujo ${ }^{1}$
}

\begin{abstract}
${ }^{1}$ Necrophagous Insects Research Group, Departamento de Zoologia, Universidade Federal de Pernambuco. Av. Prof. Moraes Rego, s/n, 50670-420 Recife-PE, Brazil.simao@ufpe.br; simaovasconcelos@yahoo.com.br

${ }^{2}$ Corresponding author
\end{abstract}

\begin{abstract}
Necrophagous species of Diptera and Coleoptera in northeastern Brazil: state of the art and challenges for the Forensic Entomologist. Inventories on necrophagous insects carried out in Brazil encompass mostly species from the southeastern and central-western regions of the country. This review aims to produce the first checklist of necrophagous Diptera and Coleoptera species of forensic relevance in northeastern Brazil, an area that concentrates high rates of homicides. We performed a literature survey on scientific articles, theses and dissertations regarding necrophagous insect species in the region, and contacted scientists who develop research on forensic entomology. Fifty-two species of Diptera belonging to eight families with previous record of necrophagy were reported in the region: Sarcophagidae, Calliphoridae, Muscidae, Fanniidae, Piophilidae, Phoridae, Anthomyiidae and Stratiomyidae. Coleopteran species from six families of forensic relevance were registered, although taxonomical identification remained superficial. Bait traps were the most frequent methodology used, followed by collection on animal carcasses. Seven Dipteran species from two families were registered on human cadavers. All species had been previously reported in other Brazilian states and/or other countries, although none has been effectively used in legal procedures in the region. The status of research on forensic entomology in northeastern Brazil is incipient, and the checklist produced here contributes to the knowledge on the local diversity of necrophagous insects.
\end{abstract}

KEYWORDS. Biodiversity; blowflies; flesh flies, forensic entomology; legal medicine.

RESUMO. Espécies necrófagas de Diptera e Coleoptera na Região Nordeste do Brasil: estado da arte e desafios para o Entomologista Forense. Inventários de insetos necrófagos no Brasil abordam em sua maioria espécies das regiões Sudeste e Centro-Oeste do país. Esta revisão visa apresentar um checklist de espécies necrófagas de Diptera e Coleoptera de importância forense no Nordeste brasileiro, uma área que concentra elevadas taxas de homicídio. Nós conduzimos uma revisão bibliográfica consultando artigos, teses e dissertações sobre espécies necrófagas na região, e contatamos cientistas que desenvolvem pesquisa em entomologia forense. Cinquenta e duas espécies de Diptera pertencentes a oito famílias com registro prévio de necrofagia foram registrados na região: Sarcophagidae, Calliphoridae, Muscidae, Fanniidae, Piophilidae, Phoridae, Anthomyiidae e Stratiomyidae. Espécies de Coleoptera de seis famílias de importância forense foram listadas, embora a identificação taxonômica tenha sido superficial. Armadilhas contendo iscas foram o método de coleta mais frequente, seguidas de coleta sobre carcaças animais. Sete espécies de Diptera pertencentes a duas famílias foram registradas sobre cadáveres humanos. Todas as espécies haviam sido previamente registradas em outros estados brasileiros e/ou em outros países, embora nenhuma tenha sido efetivamente utilizada em procedimentos legais na região. O status da pesquisa em entomologia forense no Nordeste do Brasil é incipiente, e o checklist apresentado neste trabalho contribui para sistematizar o conhecimento sobre a diversidade local de insetos necrófagos.

PALAVRAS-CHAVE. Biodiversidade; califorídeos; entomologia forense, medicina legal, sarcofagídeos.

The rate of homicides in Brazil is extremely high - and has increased 259\% between 1980 and 2010, reaching an average rate of 26.2/100,000 inhabitants (Waiselfisz 2011). Homicides are far more frequent in the nine states that comprise the Northeast region, which is partially a reflex of the region's lower social and economic development compared to the rest the country. Of the eight state capitals with the highest rate of homicides, five are located in the Northeastern region: Maceió, João Pessoa, Salvador, Recife and São Luís, all of which have homicides rates above 46,6/100,000 inhabitants (Waiselfisz 2011).

The vast majority of homicides in Northeastern Brazil is unsolved, partly due to convoluted mechanisms in the Brazilian legal system and to the deficient investment in intelligence procedures for the police modus operandi. In this context, recent advances in applied biology have prompted the need to incorporate genetic, molecular, ecological and entomological tools in the forensic scientist's routine. The consolidation of forensic entomology research in Brazil has occurred in the last two decades, and a major body of theoretical and practical work starts to become available (see Pujol-Luz et al. 2008 for a review). Such advance, however, has been limited to the capital, Brasilia and to South-Southern states. Ironically, cities that suffer from the highest rates of violence have been neglected in field inventories of forensically important species.

A variety of necrophagous insect species occurs on or around a cadaver and, depending on their preference for a given stage of decomposition, a certain chronological sequence of colonization is supposed to take place (Byrd \& 
Castner 2010). Species from the orders Diptera (e.g., Calliphoridae, Muscidae, Sarcophagidae, Stratiomyidae) and Coleoptera (e.g., Dermestidae, Cleridae, Silphidae) have been associated with criminal investigations that extrapolate their use in estimating the post-mortem interval (or period of insect activity, as suggested by Tomberlin et al. 2011). Nowadays, entomological evidence in medico-legal procedures can be used in studies of postmortal transfer, entomotoxicology, neglect of living people, association of a suspect with a victim by detection of human DNA in insects and even sexual crimes (Byrd \& Castner 2010; Tomberlin et al. 2011). None of this utility can be validated without proper knowledge on insects' bionomics, behaviour and geographical distribution.

Studies on the diversity of forensically important species have been performed by several research groups in Brazil so that an overview of native and invasive species is being compiled. The northeast region, with an area of 1,558,296 $\mathrm{km}^{2}$ and population of 55 million people still remains a major challenge for entomologists. This review aims to provide a state of the art on the occurrence of necrophagous species of Diptera and Coleoptera in the Northeast region of Brazil, according to their potential use in forensic investigations. Specifically, we aimed to: $i$ ) perform an updated literature survey, listing the most frequent species and the types of environments in which they occur; ii) examine the methodologies used in field surveys, iii) identify species that have been previously associated with medico-legal practices and iv) draw perspectives on the status of the scientific research and on the potential of forensic entomology as a routine procedure in the region.

\section{MATERIAL AND METHODS}

This review is based on a thorough bibliographical survey performed between July/2009 and September/2010 on databases from Web of Science ${ }^{\circledR}$, PubMed, Google Scholar, Periodicos Capes (a Brazilian catalogue that provides access to articles from over 15,000 scientific journals and 130 databases) and the Scientific Electronic Library Online (SciELO), which covers Latin American scientific journals, with emphasis on Natural Sciences. We also scrutinized theses and dissertations from Brazilian universities through the Ministry of Education database. Key words used in this search included general terms and scientific names related to forensic entomology (e.g., post-mortem interval, Necrobia, Chrysomya, Calliphoridae), adding geographical references to Northeastern Brazil.

Additionally, we searched for scientists who work on forensic entomology in the region, by browsing the Curriculo Lattes database, maintained by the Ministry of Science and Technology, which comprises virtually all personnel involved in scientific research in Brazil. This search retrieved the names of 36 scientists who were contacted by e-mail and asked to send their publications related to necrophagous insects.

Nine sources of data, which comprised scientific articles and M.Sc. dissertations were used, as they were available in printed or electronic version. The scientific production regarding necrophagous insect species was analyzed under the following criteria: insect taxonomical position, substrate involved (baits, animal carcasses, human cadavers) and type of environment (urban zone, forest, etc.).

\section{RESULTS AND DISCUSSION}

Overall number of families and species. Diptera species belonging to 22 families were registered. From these, species from the families Asillidae, Dixidae, Drosophilidae, Micropezidae, Tabanidae, Chloropidae, Neriidae, Milichiidae, Ropalomeridae and Tachinidae were considered to be of minor or no medico-legal importance and are not listed here. Three families (Ulidiidae, Syrphidae and Sepsidae) that, depending on the literature, can be regarded as forensically important were reported but since only one unidentified species of each family was registered, they were also excluded.

Fifty-two species from eight families of medico-legal relevance identified at least on genus level are listed in Table I. Clearly, Sarcophagidae (flesh flies), Muscidae and Calliphoridae (blowflies) comprise the highest number of species, which is a reflex of their high richness and their frequent use of decomposing animal matter as a site for feeding, mating and egg-laying (Byrd \& Castner 2010). Muscidae is a diverse family with around 5,000 species distributed throughout the world, particularly in the Neotropics, and an intimate evolutionary association with necrophagy has been established (de Carvalho et al. 2005). Calliphoridae has 190 genera and over 1,300 species described worldwide; their feeding habits include variations on parasitism, necrophagy and saprophagy (Zumpt 1965). Sarcophagidae comprises $c a$. 2,600 species distributed in all geographical regions, especially in areas with tropical climate; Neotropical sarcophagids include over 800 species (Shewell 1987; Pape 1996). Several species act as ectoparasites and their forensic importance derives from their habit of colonizing vertebrate carcasses, including man (Byrd \& Castner 2010).

When data from all surveys are combined, Coleoptera species belonging to 22 families were reported. Species from families of minor or null medico-legal importance such as Bostrichidae, Bruchidae, Chrysomelidae, Cerambycidae, Coccinellidae, Curculionidae, Elateridae and Tenebrionidae are not listed (Table II). Species from six families associated with cadavers were identified at least at genus level, from which Scarabaeidae and Histeridae were the richest families, with ten and eight species each, respectively.

The superficial taxonomical identification of Coleoptera hinders inferences about their feeding habits but it is known that species from the six families listed here (Table II) are regarded, to some extent, as forensically important even if their representatives are not obligatory necrophagous. Only ten species of necrophagous or copronecrophagous Coleoptera were fully identified: Ateuchus carbonarius (Harold, 1868), Coprophanaeus ensifer (Germar, 1824), C. jasius (Olivier, 1789), C. pertyi (d'Olsoufieff, 1924), Dichotomius 
Table I. Species of Diptera collected in field surveys of necrophagous insects in northeastern Brazil, according to the type of environment. $U=$ urban area; $\mathrm{L}=$ littoral; $\mathrm{F}=$ forest; $\mathrm{P}=$ sugarcane plantation.

\begin{tabular}{|c|c|c|c|c|}
\hline \multirow{2}{*}{ Species } & \multicolumn{4}{|c|}{ Environment } \\
\hline & $\mathrm{U}$ & $\mathrm{L}$ & $\mathrm{F}$ & $P$ \\
\hline \multicolumn{5}{|l|}{ Sarcophagidae } \\
\hline Hystricocnema plinthopyga (Wiedemann, 1830) & - & - & $\mathrm{x}$ & - \\
\hline Oxysarcodexia avuncula (Lopes, 1933) & - & - & $\mathrm{x}$ & - \\
\hline Oxysarcodexia fluminensis Lopes, 1946 & - & - & $\mathrm{X}$ & - \\
\hline Oxysarcodexia intona (Curran \& Walley, 1934) & - & - & $\mathrm{x}$ & - \\
\hline Oxysarcodexia modesta Lopes, 1946 & $\mathrm{x}$ & - & $\mathrm{x}$ & - \\
\hline Oxysarcodexia riograndensis Lopes, 1946 & $\mathrm{x}$ & - & $\mathrm{x}$ & - \\
\hline Oxysarcodexia simplicoides Lopes, 1933 & $\mathrm{x}$ & - & - & - \\
\hline Oxysarcodexia thornax (Wiedemann, 1830) & $\mathrm{x}$ & - & - & - \\
\hline Oxyvinia excisa (Lopes, 1950) & - & - & $\mathrm{x}$ & - \\
\hline Peckia (Peckia) chrysostoma (Wiedemann, 1830) & - & $\mathrm{x}$ & - & _- \\
\hline Peckia (Pattonella) intermutans (Walker, 1861) & $\mathrm{x}$ & - & $\mathrm{X}$ & - \\
\hline Peckia (Squamatodes) ingens (Walker, 1849) & $\mathrm{x}$ & $\mathrm{x}$ & $\mathrm{x}$ & - \\
\hline Ravinia belforti (Prado \& Fonseca, 1932) & $\mathrm{x}$ & _ & $\mathrm{x}$ & $\mathrm{x}$ \\
\hline Sarcodexia lambens (Wiedemann, 1830) & $\mathrm{x}$ & - & - & - \\
\hline Tricharaea (Sarcophagula) occidua (Fabricius, 1794) & - & $\mathrm{x}$ & - & - \\
\hline Tricharaea sp. & $\mathrm{x}$ & $\mathrm{X}$ & $\mathrm{x}$ & $\mathrm{X}$ \\
\hline \multicolumn{5}{|l|}{ Muscidae } \\
\hline Atherigona orientalis Schiner, 1868 & $\mathrm{x}$ & $\mathrm{x}$ & $\mathrm{x}$ & $\mathrm{X}$ \\
\hline Biopyrellia bipuncta (Wiedemann, 1830) & $\mathrm{x}$ & _ & _- & - \\
\hline Brontaea delecta (Wulp, 1896) & $\mathrm{x}$ & - & - & - \\
\hline Brontaea normata (Bigot, 1885) & $\mathrm{x}$ & - & $\mathrm{x}$ & - \\
\hline Cariocamyia maculosa Snyder, 1951 & $\mathrm{x}$ & _- & $\mathrm{x}$ & _ \\
\hline Cyrtoneuropsis rescita (Walker, 1861) & - & $\mathrm{x}$ & - & - \\
\hline Graphomya analis (Macquart, 1851) & $\mathrm{x}$ & - & - & - \\
\hline Graphomya maculata (Scopoli, 1763) & - & - & $\mathrm{x}$ & - \\
\hline Hydrotaea nicholsoni Curran, 1939 & $\mathrm{x}$ & - & - & - \\
\hline Morellia humeralis (Stein, 1898) & - & - & $\mathrm{x}$ & - \\
\hline Musca domestica Linnaeus, 1758 & $\mathrm{x}$ & $\mathrm{x}$ & $\mathrm{x}$ & $\mathrm{x}$ \\
\hline Ophyra aenescens (Wiedemann, 1830) & $\mathrm{x}$ & - & - & - \\
\hline Ophyra chalcogaster (Wiedemann, 1824) & $\mathrm{x}$ & $\mathrm{x}$ & $\mathrm{x}$ & - \\
\hline Parapyrellia maculipennis (Macquart, 1846) & _- & _- & $\mathrm{x}$ & - \\
\hline Synthesiomyia nudiseta (Wulp, 1883) & $\mathrm{x}$ & $\mathrm{x}$ & - & $\mathrm{X}$ \\
\hline \multicolumn{5}{|l|}{ Calliphoridae } \\
\hline Cochliomyia macellaria (Fabricius, 1775) & $\mathrm{x}$ & $\mathrm{x}$ & $\mathrm{x}$ & $\mathrm{X}$ \\
\hline Chloroprocta idioidea (Robineau-Desvoidy, 1830) & - & - & $\mathrm{x}$ & - \\
\hline Chrysomya albiceps (Wiedemann, 1819) & $\mathrm{x}$ & $\mathrm{x}$ & $\mathrm{x}$ & $\mathrm{X}$ \\
\hline Chrysomya megacephala (Fabricius, 1794) & $\mathrm{x}$ & $\mathrm{x}$ & $\mathrm{x}$ & $\mathrm{X}$ \\
\hline Chrysomya putoria (Wiedemann, 1819) & $\mathrm{x}$ & $\mathrm{x}$ & $\mathrm{X}$ & $\mathrm{X}$ \\
\hline Hemilucilia segmentaria (Fabricius, 1805) & $\mathrm{x}$ & - & $\mathrm{x}$ & - \\
\hline Hemilucilia semidiaphana (Rondani, 1850) & - & - & $\mathrm{x}$ & - \\
\hline Lucilia cuprina (Wiedemann, 1830) & $\mathrm{x}$ & - & - & - \\
\hline Lucilia eximia (Wiedemann, 1819) & $\mathrm{x}$ & $\mathrm{x}$ & $\mathrm{x}$ & $\mathrm{X}$ \\
\hline Lucilia sericata (Meigen, 1826) & - & _- & $\mathrm{x}$ & _- \\
\hline Mesembrinella bellardiana (Séguy, 1925) & - & - & $\mathrm{x}$ & - \\
\hline \multicolumn{5}{|l|}{ Fanniidae } \\
\hline Fannia canicularis (Linnaeus, 1761) & $\mathrm{x}$ & $\mathrm{x}$ & $\mathrm{x}$ & _- \\
\hline Fannia obscurinervis (Stein, 1900) & - & - & $\mathrm{X}$ & - \\
\hline Fannia pusio (Wiedemann, 1830) & $\mathrm{x}$ & $\mathrm{x}$ & $\mathrm{x}$ & $\mathrm{X}$ \\
\hline \multicolumn{5}{|l|}{ Anthomyiidae } \\
\hline Anthomyia aurifacies (Albuquerque, 1952) & - & - & $\mathrm{x}$ & - \\
\hline Anthomyia plurinervis (Albuquerque, 1958) & - & - & $\mathrm{x}$ & - \\
\hline Anthomyia punctipennis (Wiedemann, 1830) & - & - & $\mathrm{x}$ & - \\
\hline \multicolumn{5}{|l|}{ Piophilidae } \\
\hline Piophila casei (Linnaeus, 1758) & $\mathrm{x}$ & $\mathrm{x}$ & $\mathrm{x}$ & $\mathrm{x}$ \\
\hline Piophila sp. & - & - & $\begin{array}{c}\mathrm{X} \\
\text { Cont }\end{array}$ & $\begin{array}{c}- \\
\text { nue }\end{array}$ \\
\hline
\end{tabular}

Table I. Continued.

\begin{tabular}{|c|c|c|c|c|}
\hline \multirow{2}{*}{ Species } & \multicolumn{4}{|c|}{ Environment } \\
\hline & $\mathrm{U}$ & $\mathrm{L}$ & $\mathrm{F}$ & $\mathrm{P}$ \\
\hline \multicolumn{5}{|l|}{ Phoridae } \\
\hline Megaselia scalaris (Loew, 1866) & $\mathrm{X}$ & $\mathrm{X}$ & $\mathrm{X}$ & $\mathrm{X}$ \\
\hline \multicolumn{5}{|l|}{ Stratiomyidae } \\
\hline Hermetia illucens (Linnaeus, 1758) & $\mathrm{X}$ & - & $\mathrm{X}$ & - \\
\hline
\end{tabular}

Additional sources: Andrade et al. (2005); Nilo (2007); Couri et al. (2008); Cruz (2008); Oliveira \& Vasconcelos (2010); A. R. B. Santos (pers. comm.).

risus Olivier, 1789, Digitonthophagus gazella (Fabricius, 1787), Omalodes bifoveolatus Marseul, 1853, Dermestes maculatus (De Geer, 1774), Xanthopygus bicolor (LaPorte, 1835) and Necrobia rufipes (De Geer, 1775) (Table II).

Table II. Species of Coleoptera of forensic importance collected in field experiments in northeastern Brazil, according to the environment ( $\mathrm{U}=$ urban area; $\mathrm{L}=$ littoral; $\mathrm{F}=$ forest $\mathrm{C}=$ caatinga).

\begin{tabular}{|c|c|c|c|c|}
\hline \multirow{2}{*}{ Species } & \multicolumn{4}{|c|}{ Environment } \\
\hline & $\mathrm{U}$ & $\mathrm{L}$ & $\mathrm{F}$ & $\mathrm{C}$ \\
\hline \multicolumn{5}{|l|}{ Scarabaeidae } \\
\hline Ateuchus carbonarius (Harold, 1868) & - & - & $\mathrm{X}$ & $\mathrm{X}$ \\
\hline Canthon sp. & - & - & $\mathrm{X}$ & - \\
\hline Coprophanaeus ensifer (Germar,1824) & - & - & $\mathrm{X}$ & - \\
\hline Coprophanaeus jasius (Olivier, 1789) & - & - & - & $\mathrm{X}$ \\
\hline Coprophanaeus pertyi (d'Olsoufieff, 1924) & - & - & - & $\mathrm{X}$ \\
\hline Coprophanaeus sp. & - & - & $\mathrm{X}$ & - \\
\hline Delthochilum sp. & $\mathrm{X}$ & $\mathrm{X}$ & $\mathrm{X}$ & - \\
\hline Dichotomius nisus Olivier, 1789 & - & - & $\mathrm{X}$ & $\mathrm{X}$ \\
\hline Dichotomius sp. & - & - & $\mathrm{X}$ & - \\
\hline Digitonthophagus gazella (Fabricius, 1787) & - & - & $\mathrm{X}$ & $\mathrm{X}$ \\
\hline \multicolumn{5}{|l|}{ Histeridae } \\
\hline Euspilotus sp. & - & - & $\mathrm{X}$ & - \\
\hline Hister sp. & $\mathrm{X}$ & $\mathrm{X}$ & $\mathrm{X}$ & - \\
\hline Holepta sp. & - & - & $\mathrm{X}$ & - \\
\hline Phelister sp. & - & - & $\mathrm{X}$ & - \\
\hline Omalodes bifoveolatus Marseul, 1853 & - & - & $\mathrm{X}$ & - \\
\hline Omalodes sp. & $\mathrm{X}$ & $\mathrm{X}$ & $\mathrm{X}$ & - \\
\hline Saprinus sp. & - & - & $\mathrm{X}$ & - \\
\hline Xestipyge sp. & - & - & $\mathrm{X}$ & - \\
\hline \multicolumn{5}{|l|}{ Dermestidae } \\
\hline Dermestes maculatus (De Geer, 1774) & $\mathrm{X}$ & $\mathrm{X}$ & $\mathrm{X}$ & $X$ \\
\hline \multicolumn{5}{|l|}{ Staphylinidae } \\
\hline Aleochara sp. & - & - & $\mathrm{X}$ & - \\
\hline Atheta sp. & - & - & $\mathrm{X}$ & $\mathrm{X}$ \\
\hline Belonchus sp. & - & - & $\mathrm{X}$ & - \\
\hline Philonthus sp. & - & - & $\mathrm{X}$ & - \\
\hline Xanthopygus bicolor (LaPorte, 1835) & - & - & $\mathrm{X}$ & - \\
\hline \multicolumn{5}{|l|}{ Trogidae } \\
\hline Omorgus sp. & - & - & $\mathrm{X}$ & - \\
\hline \multicolumn{5}{|l|}{ Cleridae } \\
\hline Necrobia rufipes (De Geer,1775) & $X$ & $X$ & $X$ & $X$ \\
\hline
\end{tabular}

Additional sources: Endres et al. (2005); Cruz \& Vasconcelos (2006); Cruz (2008); Mayer (2009); C. L. Bicho (pers. comm.).

Diversity of collection techniques. Several methods of capture and types of substrate have been used in field inventories in the region, ranging from short-term collection using meat baits and pitfall traps to non-replicated experiments 
using animal carcasses and collections on human corpses (Tables III and IV). The most frequent method was a modification of the trap designed by Ferreira (1978), which is a portable, inexpensive and effective technique that collects mainly Dipteran adults. Although suspended bait traps have been successfully used to collect necrophagous species in Brazil and abroad, their drawbacks include their non-selective nature and the rapid decomposition of animal tissues used as baits so that collection favours early colonizers and is little effective for capture of late colonizers and non-flying necrophagous beetles.

Field studies performed in Northeastern Brazil have used animal tissues as varied as fish, pork and, especially because of the high number of species collected, chicken liver. Bait traps were associated with the collection of 16 species of Sarcophagidae, seven Calliphoridae and also species of scuttle flies (Phoridae), Lesser houseflies (Fanniidae) and skipper flies (Piophilidae).

Pitfall traps scattered around animal carcasses (Mayer 2009) or containing decomposing meat and human faeces as attractants (Endres et al. 2005) were used for the collection of soil copronecrophagous coleopterans. These heterogeneous methods make it difficult to differentiate accidental species (e.g. Curculionidae, Cerambycidae) from the true necrophagous, since feeding habits can vary within one Coleoptera genus or even within different stages of development of the same species (Arnett Jr. et al. 2002). Also, the non-discriminate nature of pitfall traps cause the collection of several nonnecrophagous species, which can be affected by factors as diverse as trap quantity, size and spacing, time of exposure and type of bait (Ward et al. 2001).

Carcasses varied from pigs and dogs to hamsters, rats and rabbits. Domestic pigs have been successfully used as models in forensic entomology for decades, as there are similarities with the dermal composition, gut flora and rates of tissue decomposition when compared to humans (Catts \& Goff 1992). The higher number of dipteran and coleopteran species associated with pig carcasses (43 spp.) reflects the reliability of this model which is, in turn, used more frequently than other vertebrates.

Insect collection on carcasses involved mostly flight interception traps for dipterans (e.g., Cruz 2008), direct collection of larvae and adults on the carcass (especially for Coleoptera) and the placement of trays containing sawdust under the carcass to collect immature and adult coleopterans and also dipteran larvae at the post-feeding stage.

In the region, only two studies (Andrade et al. 2005; Oliveira \& Vasconcelos 2010) analysed insect fauna from corpses, increasing the applicability of the data. Combined data from those studies totalled seven species from two families: Oxysarcodexia riograndensis Lopes, 1943, Ravinia belforti (Prado \& Fonseca, 1932) (Sarcophagidae), Chrysomya albiceps (Wiedemann, 1819), C. megacephala (Fabricius, 1794), Cochliomyia macellaria (Fabricius, 1775), Lucilia cuprina (Wiedemann, 1830) and L. eximia (Wiedemann, 1819) (Calliphoridae) (Table III).
Table III. Species of Diptera collected in field experiments on forensic entomology in northeastern Brazil, according to the type of substrate: bait $(\mathrm{C}=$ chicken liver; $\mathrm{S}=$ sardine, $\mathrm{P}=$ pork $)$, carcass $(\mathrm{Pi}=$ pig; $\mathrm{D}=\operatorname{dog} ; \mathrm{R}$ $=$ rodent; $\mathrm{Rb}=$ rabbit) or cadaver.

\begin{tabular}{|c|c|c|c|c|c|c|c|}
\hline \multirow{2}{*}{ Species } & \multicolumn{3}{|c|}{ Bait } & \multicolumn{3}{|c|}{ Carcass } & \multirow{2}{*}{ Cadaver } \\
\hline & $\mathrm{C}$ & 5 & $P$ & $\mathrm{Pi}$ & D & $\mathrm{Rb}$ & \\
\hline
\end{tabular}

\begin{tabular}{|c|c|c|c|c|c|c|c|c|}
\hline \multicolumn{9}{|l|}{ Sarcophagidae } \\
\hline Hystricocnema plinthopyga & $\mathrm{X}$ & - & - & $\mathrm{X}$ & - & $\mathrm{X}$ & - & - \\
\hline Oxysarcodexia avuncula & - & - & - & $\mathrm{X}$ & - & - & - & - \\
\hline Oxysarcodexia fluminensis & - & - & - & $\mathrm{X}$ & - & - & - & - \\
\hline Oxysarcodexia intona & - & - & - & $\mathrm{X}$ & - & - & - & - \\
\hline Oxysarcodexia modesta & $\mathrm{X}$ & - & - & $\mathrm{X}$ & - & - & - & - \\
\hline Oxysarcodexia riograndensis & $\mathrm{X}$ & - & - & $\mathrm{X}$ & - & - & $\mathrm{X}$ & $\mathrm{X}$ \\
\hline Oxysarcodexia simplicoides & - & - & - & - & - & $\mathrm{X}$ & - & - \\
\hline Oxysarcodexia thornax & - & - & - & - & - & $\mathrm{X}$ & - & - \\
\hline Oxyvinia excisa & - & - & - & $\mathrm{X}$ & - & - & - & - \\
\hline Peckia(Peckia) chrysostoma & $\mathrm{X}$ & $\mathrm{X}$ & - & $\mathrm{X}$ & - & - & - & - \\
\hline Peckia (Pattonella) intermutans & $\mathrm{X}$ & - & - & - & - & $\mathrm{X}$ & - & - \\
\hline Peckia (Squamatodes) ingens & $\mathrm{X}$ & - & - & $\mathrm{X}$ & - & $\mathrm{X}$ & $\mathrm{X}$ & - \\
\hline Ravinia belforti & $\mathrm{X}$ & $\mathrm{X}$ & $\mathrm{X}$ & - & - & - & $\mathrm{X}$ & $\mathrm{X}$ \\
\hline Sarcodexia lambens & $\mathrm{X}$ & $\mathrm{X}$ & - & - & - & - & $\mathrm{X}$ & - \\
\hline Tricharaea (Sarcophagula) occidua & $\mathrm{X}$ & - & - & - & - & - & - & - \\
\hline Tricharaea sp. & $\mathrm{X}$ & $\mathrm{X}$ & $\mathrm{X}$ & $\mathrm{X}$ & - & - & - & - \\
\hline \multicolumn{9}{|l|}{ Muscidae } \\
\hline Atherigona orientalis & $\mathrm{X}$ & $\mathrm{X}$ & $\mathrm{X}$ & $\mathrm{X}$ & - & - & - & - \\
\hline Biopyrellia bipuncta & $\mathrm{X}$ & - & - & $\mathrm{X}$ & - & - & - & - \\
\hline Brontaea delecta & $\mathrm{X}$ & - & - & - & - & $\mathrm{X}$ & - & - \\
\hline Brontaea normata & $\mathrm{X}$ & - & - & $\mathrm{X}$ & - & - & - & - \\
\hline Cariocamyia maculosa & $\mathrm{X}$ & $\mathrm{X}$ & - & - & - & - & - & - \\
\hline Cyrtoneuropsis rescita & $\mathrm{X}$ & - & - & - & - & - & - & - \\
\hline Graphomya analis & $\mathrm{X}$ & $\mathrm{X}$ & - & - & - & - & - & - \\
\hline Graphomya maculate & - & - & - & - & $\mathrm{X}$ & - & - & - \\
\hline Hydrotaea nicholsoni & $\mathrm{X}$ & - & - & $\mathrm{X}$ & - & - & - & - \\
\hline Musca domestica & $\mathrm{X}$ & $\mathrm{X}$ & $\mathrm{X}$ & $\mathrm{X}$ & - & $\mathrm{X}$ & - & - \\
\hline Morellia humeralis & - & - & - & $\mathrm{X}$ & - & - & - & - \\
\hline Ophyra aenescens & $\mathrm{X}$ & $\mathrm{X}$ & - & - & - & - & - & - \\
\hline Ophyra chalcogaster & $\mathrm{X}$ & $\mathrm{X}$ & - & $\mathrm{X}$ & - & $\mathrm{X}$ & - & - \\
\hline Parapyrellia maculipennis & - & - & - & $\mathrm{X}$ & - & - & - & - \\
\hline Synthesiomyia nudiseta & $\mathrm{X}$ & $\mathrm{X}$ & $\mathrm{X}$ & $\mathrm{X}$ & - & - & - & - \\
\hline \multicolumn{9}{|l|}{ Calliphoridae } \\
\hline Cochliomyia macellaria & - & $\mathrm{X}$ & $\mathrm{X}$ & $\mathrm{X}$ & - & $\mathrm{X}$ & - & $\mathrm{X}$ \\
\hline Chloroprocta idioidea & - & - & $\mathrm{X}$ & $\mathrm{X}$ & - & - & - & - \\
\hline Chrysomya albiceps & $\mathrm{X}$ & $\mathrm{X}$ & $\mathrm{X}$ & $\mathrm{X}$ & $\mathrm{X}$ & $\mathrm{X}$ & $\mathrm{X}$ & $\mathrm{X}$ \\
\hline Chrysomya megacephala & $\mathrm{X}$ & $\mathrm{X}$ & $\mathrm{X}$ & $\mathrm{X}$ & $\mathrm{X}$ & $\mathrm{X}$ & $\mathrm{X}$ & $\mathrm{X}$ \\
\hline Chrysomya putoria & $\mathrm{X}$ & $\mathrm{X}$ & $\mathrm{X}$ & $\mathrm{X}$ & $\mathrm{X}$ & $\mathrm{X}$ & $\mathrm{X}$ & - \\
\hline Hemilucilia segmentaria & $\mathrm{X}$ & - & - & $\mathrm{X}$ & $\mathrm{X}$ & $\mathrm{X}$ & - & - \\
\hline Hemilucilia semidiaphana & & - & - & $\mathrm{X}$ & - & - & - & - \\
\hline Lucilia cuprina & - & - & - & - & $\mathrm{X}$ & - & - & $\mathrm{X}$ \\
\hline Lucilia eximia & $\mathrm{X}$ & $\mathrm{X}$ & $\mathrm{X}$ & $\mathrm{X}$ & $\mathrm{X}$ & $\mathrm{X}$ & - & $\mathrm{X}$ \\
\hline Lucilia sericata & - & - & - & - & $\mathrm{X}$ & - & - & - \\
\hline Mesembrinella bellardiana & - & $\mathrm{X}$ & $\mathrm{X}$ & $\mathrm{X}$ & - & - & - & - \\
\hline \multicolumn{9}{|l|}{ Fanniidae } \\
\hline Fannia canicularis & $\mathrm{X}$ & - & - & - & - & $\mathrm{X}$ & - & - \\
\hline Fannia obscurinervis & - & - & - & $\mathrm{X}$ & - & - & - & - \\
\hline Fannia pusio & $\mathrm{X}$ & $\mathrm{X}$ & $\mathrm{X}$ & - & - & - & - & - \\
\hline \multicolumn{9}{|l|}{ Anthomyiidae } \\
\hline Anthomyia aureficies & - & - & - & $\mathrm{X}$ & - & - & - & - \\
\hline Anthomyia plurinervis & - & - & - & $\mathrm{X}$ & - & - & - & - \\
\hline Anthomyia punctipennis & - & - & - & $\mathrm{X}$ & - & - & - & - \\
\hline \multicolumn{9}{|l|}{ Piophilidae } \\
\hline Piophila casei & $\mathrm{X}$ & $\mathrm{X}$ & $\mathrm{X}$ & $\mathrm{X}$ & $\mathrm{X}$ & $\mathrm{X}$ & - & - \\
\hline Piophila sp. & - & - & - & $\mathrm{X}$ & - & - & - & - \\
\hline
\end{tabular}


Table III. Continued.

\begin{tabular}{|c|c|c|c|c|c|c|c|c|}
\hline \multirow{2}{*}{ Species } & \multicolumn{3}{|c|}{ Bait } & \multicolumn{4}{|c|}{ Carcass } & \multirow{2}{*}{ Cadaver } \\
\hline & C & S & $\mathrm{P}$ & $\mathrm{Pi}$ & D & $\mathrm{R}$ & $\mathrm{Rb}$ & \\
\hline \multicolumn{9}{|l|}{ Phoridae } \\
\hline Megaselia scalaris & $\mathrm{X}$ & $\mathrm{X}$ & $\mathrm{X}$ & $\mathrm{X}$ & $\mathrm{X}$ & - & - & - \\
\hline \multicolumn{9}{|l|}{ Stratiomyidae } \\
\hline Hermetia illucens & $X$ & - & - & $X$ & X & X & - & - \\
\hline
\end{tabular}

Table IV. Species of Coleoptera collected in field experiments on forensic entomology in Northeastern Brazil, according to the type of carcass $(\mathrm{P}=$ pig; $\mathrm{D}=\operatorname{dog} ; \mathrm{R}=$ rodent, $\mathrm{Rb}=$ rabbit) and previous report of forensic importance for the family in the literature.

\begin{tabular}{|c|c|c|c|c|c|}
\hline \multirow{2}{*}{ Species } & \multirow{2}{*}{ Forensic importance } & \multicolumn{4}{|c|}{ Type of carcass } \\
\hline & & $\mathrm{P}$ & $\mathrm{D}$ & $\mathrm{R}$ & $\mathrm{Rb}$ \\
\hline Histeridae & $\mathrm{a}, \mathrm{b}, \mathrm{c}, \mathrm{d}, \mathrm{e}$ & & & & \\
\hline Euspilotus sp. & & $\mathrm{X}$ & $\mathrm{X}$ & - & $\mathrm{X}$ \\
\hline Hister sp. & & - & $\mathrm{X}$ & $\mathrm{X}$ & $\mathrm{X}$ \\
\hline Holepta sp. & & - & $\mathrm{X}$ & - & - \\
\hline Omolodes bifoveolatus & & - & - & - & $\mathrm{X}$ \\
\hline Omalodes sp. & & $\mathrm{X}$ & $\mathrm{X}$ & $\mathrm{X}$ & - \\
\hline Phelister $\mathrm{sp}$. & & - & - & - & $\mathrm{X}$ \\
\hline Saprinus sp. & & - & - & - & $\mathrm{X}$ \\
\hline Xestipyge sp. & & $\mathrm{X}$ & - & - & - \\
\hline Scarabaeidae & $\mathrm{a}, \mathrm{b}, \mathrm{c}, \mathrm{d}, \mathrm{e}$ & & & & \\
\hline Ateuchus carbonarius* & & - & - & - & - \\
\hline Canthon sp. & & $\mathrm{X}$ & - & - & - \\
\hline Coprophanaeus ensifer* & & - & - & - & - \\
\hline Coprophanaeus jasius* & & - & - & - & - \\
\hline Coprophanaeus pertyi* & & - & - & - & - \\
\hline Coprophanaeus sp. & & $\mathrm{X}$ & - & - & - \\
\hline Delthochilum sp.* & & - & - & - & - \\
\hline Dichotomius nisus* & & - & - & - & - \\
\hline Dichotomius sp.* & & $\mathrm{X}$ & - & - & - \\
\hline Digitonthophagus gazella* & & - & - & - & - \\
\hline Dermestidae & $\mathrm{a}, \mathrm{b}, \mathrm{c}, \mathrm{d}, \mathrm{e}, \mathrm{f}, \mathrm{g}, \mathrm{i}$ & & & & \\
\hline Dermestes maculatus & & $\mathrm{X}$ & - & $\mathrm{X}$ & - \\
\hline Staphylinidae & $\mathrm{a}, \mathrm{b}, \mathrm{c}, \mathrm{d}, \mathrm{e}, \mathrm{h}$ & & & & \\
\hline Aleochara sp. & & - & - & - & $\mathrm{X}$ \\
\hline Atheta sp.* & & - & - & - & - \\
\hline Belonchus sp. & & - & - & - & $\mathrm{X}$ \\
\hline Philonthus sp. & & - & - & - & $\mathrm{X}$ \\
\hline Xanthopygus bicolour & & - & - & - & $\mathrm{X}$ \\
\hline Trogidae & $\mathrm{a}, \mathrm{b}, \mathrm{e}$ & & & & \\
\hline Omorgus sp. & & $\mathrm{X}$ & - & - & - \\
\hline Cleridae & $a, b, c, d, e, f, g$ & & & & \\
\hline Necrobia rufipes & & $X$ & - & $X$ & - \\
\hline
\end{tabular}

* Species found exclusively on baited pitfall traps. Sources: $a=$ Byrd \& Castner (2010); b = Oliveira-Costa (2011); c = Smith (1986); d = Carvalho et al. (2000); e = Almeida \& Mise (2009); $\mathrm{f}=$ Schroeder et al. (2002); $\mathrm{g}=$ Kulshrestha \& Satpathy (2001); h = Marquez-Luna (2001); i = Sukontason et al. (2007).

Regardless of their abundance, insect collection on animal carcasses does not necessarily mean necrophagy: because carcasses are ephemeral resources rapidly colonized by thousands of immature and adult individuals, they are a suitable feeding site for predators and parasitoids in search of hosts. For example, from the 32 dipteran species collected flying over a pig carcass in a forest remnant in Recife, Pernambuco, only five effectively used the resource to complete their life cycle (Vasconcelos, S. D., unpublished data). The high diversity of Histeridae (Table II), a predatory coleopteran family, reinforces the ubiquity of non-necrophagous species associated with decomposing bodies.

Geographical distribution and types of environment. The variety of biomes, ecosystems and environment has been only superficially explored in the inventories. The northeast region encompasses rain forests, a long coastline ( $c a$. 3,300 $\mathrm{km}$ ), dunes, canyons, mangroves, caatinga (a type of dry forest endemic to Brazilian territory), diversified agroecosystems, large cities, among others, and such variety should be reflected in the diversity of necrophagous species. Forest remnants have been the commonest environment used for inventories, not only for its ecological importance as habitats for a wide variety of species, but also because urban enclaves of rainforests are the most frequent repositories for cadavers resulting from homicides in large cities in the region. Thirteen species of sarcophagids, nine species of calliphorids and three of muscids were registered in forest fragments in cities such as Recife (Pernambuco State) and Salvador (Bahia State) (Table I). Necrophagous species reported exclusively in forest remnants in the region so far include Hystriocnema plinthopyga (Wiedemann, 1830), Oxysacordexia avuncular (Lopes, 1933), O. fluminensis Lopes, 1946, O. intona (Curran \& Walley, 1934), O. modesta Lopes, 1946, Oxyvinia excisa (Lopes, 1950) (Sarcophagidae), Chloroprocta idioidea ((Robineau-Desvoidy, 1830)), Hemilucilia segmentaria (Fabricius, 1805), $H$. semidiaphana (Randoni, 1850) and Lucilia sericata (Meigen, 1826) (Calliphoridae) all of which have been previously regarded as having forensic relevance (Carvalho et al. 2004). Coleopteran species found exclusively on forest fragments so far included Euspilotus sp., Holepta sp., Xestipyge sp., Omalodes bifoveolatus Marseul, 1853, Sapinus sp. (Histeridae), Coprophaneous ensifer, Coprophanaeus sp., Canthon sp., Dichotomius sp. (Scarabaeidae) and Omorgus sp. (Trogidae).

Data on necrophagous fauna in the littoral is incipient: so far, necrophagous species reported in coastal zones included four sarcophagids, four calliphorids and four muscids (Table I). Couri et al. (2008), working on material collected in the 1970's in the archipelago of Fernando de Noronha, $550 \mathrm{~km}$ from Pernambuco coastline, listed eight dipteran families, including Calliphoridae, Sarcophagidae and Muscidae. Tricharaea (Sarcophagula) occidua (Fabricius, 1794) (Sarcophagidae) was reported only in beach ecosystems (Couri et al. 2008; Oliveira 2009), similarly to what was observed in Argentina (Mariluis et al. 2007). No necrophagous coleopteran species were found to be exclusive to coastal zone according to the reviewed literature.

Urban areas have been sampled more frequently than other ecosystems, mainly due to their easy access and to the frequency of homicides. Most cases of actual use of entomological evidence (e.g., C. albiceps, C. megacephala, C. putoria and L. eximia) for estimating the PMI in other countries occurred in urban areas (Table V) and the location of 
institutes of legal medicine in state capitals increases the importance of collecting data on synanthropic species. Dipteran species with strong association with urban environments in Northeastern Brazil include: O. simplicoides Lopes, 1933, O. thornax (Walker, 1849), Sarcodexia lambens (Wiedmann, 1830) (Sarcophagidae), Biopyrellia bipuncta (Wiedemann, 1830), Brontaea delecta (Wulp, 1896), Hydrotaea nicholsoni Curran, 1939, Ophyra aenescens (Wiedemann, 1830) (Muscidae) and L. cuprina (Calliphoridae). No necrophagous coleopteran species were found exclusively in urban zones.

A few species were reported throughout several ecosystems, such as $C$. megacephala, C. albiceps, C. macellaria, $L$. eximia (Calliphoridae), Megaselia scalaris (Loew, 1866) (Phoridae), M. domestica Linnaeus, 1758 and Atherigona orientalis Schiner, 1868 (Muscidae). Naturally, this is a dynamic situation: for example, the introduction of Chrysomya species in the 1970's is believed to have dislocated native species from their habitats due mainly to predatory behaviour and larval competition (Wells \& Greenberg 1992) so that a widespread occurrence of Chrysomya species may be reported in near future.

Table V. Species of Diptera with previous register of necrophagy collected in field experiments in northeastern Brazil, according to occurrence on human cadavers and register of actual use in forensic entomology cases. Forensic importance was also based on de Carvalho \& Mello-Patiu (2008).

\begin{tabular}{|c|c|c|}
\hline Species & Human cadaver & Forensic Entomology \\
\hline \multicolumn{3}{|l|}{ Calliphoridae } \\
\hline Chrysomya albiceps & $\mathrm{k}, \mathrm{l}, \mathrm{m}, \mathrm{n}, \mathrm{o}$ & $\mathrm{n}, \mathrm{o}$ \\
\hline Chrysomya megacephala & $\mathrm{k}, \mathrm{l}, \mathrm{m}, \mathrm{n}, \mathrm{p}, \mathrm{q}, \mathrm{r}$ & $\mathrm{n}$ \\
\hline Chrysomya putoria & $\mathrm{m}$ & - \\
\hline Cochliomyia macellaria & $\mathrm{k}, 1, \mathrm{~m}, \mathrm{r}$ & - \\
\hline Hemilucilia segmentaria & $\mathrm{m}$ & $\mathrm{z}$ \\
\hline Hemilucilia semidiaphana & $\mathrm{m}$ & - \\
\hline Lucilia cuprina & $1, \mathrm{n}, \mathrm{r}$ & - \\
\hline Lucilia eximia & $1, \mathrm{~m}$ & - \\
\hline \multicolumn{3}{|l|}{ Sarcophagidae } \\
\hline Oxysarcodexia riograndensis & $\mathrm{k}$ & - \\
\hline Peckia (Pattonella) intermutans & $\mathrm{m}$ & - \\
\hline Ravinia belforti & $\mathrm{k}$ & - \\
\hline \multicolumn{3}{|l|}{ Muscidae } \\
\hline Musca domestica & $\mathrm{n}, \mathrm{q}$ & - \\
\hline Ophyra chalcogaster & $\mathrm{m}$ & - \\
\hline Synthesiomyia nudiseta & $\mathrm{n}, \mathrm{r}, \mathrm{s}$ & - \\
\hline \multicolumn{3}{|l|}{ Fanniidae } \\
\hline Fannia canicularis & $\mathrm{v}, \mathrm{n}$ & $\mathrm{v}$ \\
\hline \multicolumn{3}{|l|}{ Piophilidae } \\
\hline Piophila casei & $\mathrm{m}, \mathrm{t}, \mathrm{r}$ & $\mathrm{t}$ \\
\hline \multicolumn{3}{|l|}{ Phoridae } \\
\hline Megaselia scalaris & $\mathrm{m}, \mathrm{r}, \mathrm{y}$ & $\mathrm{w}$ \\
\hline \multicolumn{3}{|l|}{ Stratiomyidae } \\
\hline Hermetia illucens & $\mathrm{x}, \mathrm{m}, \mathrm{n}, \mathrm{u}$ & $\mathrm{x}, \mathrm{u}$ \\
\hline \multicolumn{3}{|c|}{$\begin{array}{l}\text { Sources: } \mathrm{k}=\text { Oliveira \& Vasconcelos (2010); } \mathrm{l}=\text { Andrade } \text { et al. } \text { (2005); } \mathrm{m}=\text { Carvalho } \\
\text { et al. }(2000) ; \mathrm{n}=\text { Velázquez et al. }(2010) ; \mathrm{o}=\text { Grassberger } \text { et al. } \text { (2003); } \mathrm{p}=\text { Oliveira } \\
\text {-Costa \& Mello-Patiu (2004); } \mathrm{q}=\text { Barreto } \text { et al. } \text { (2002); } \mathrm{r}=\text { Sukontason } \text { et al. } \text { (2007); } \\
\mathrm{s}=\text { Shewell (1987); } \mathrm{t}=\text { Benecke }(1998) ; \mathrm{u}=\text { Lord } \text { et al. }(1994) ; \mathrm{v}=\text { Benecke \& Lessig } \\
(2001) ; \mathrm{w}=\text { Greenberg \& Wells }(1998) ; \mathrm{x}=\text { Pujol-Luz et al. } \text { (2006); } \mathrm{y}=\text { Disney } \\
(2008) ; \mathrm{z}=\text { Kosmann } \text { et al. } \text { (2011). }\end{array}$} \\
\hline
\end{tabular}

Revista Brasileira de Entomologia 56(1): 7-14, março, 2012
The status of research on forensic entomology in the region. References on necrophagous insect species originated from six out of the nine states in the region: Pernambuco, Rio Grande do Norte, Paraiba, Bahia, Maranhao and Alagoas. All species registered in the region have been previously reported for other Brazilian states and many of them were also registered in other countries. Is it possible that further taxonomical identification may demonstrate the occurrence of new species, or first records. Most species were listed for Pernambuco, which does not necessarily imply a higher diversity but reflects the fact that forensic research has been more firmly established in that state. From the ongoing projects on the ecology of necrophagous Diptera and Coleoptera in other states, it is expected that updated lists of necrophagous species will be available in the next few years.

Research on forensic entomology is incipient in Northeastern Brazil and appears to be limited to public universities, despite the alarming rates of unsolved homicides. Contact with local scientists reinforces the notion that forensic entomology is, until now, a secondary line of investigation. The studies performed so far are isolated initiatives with limited financial support and rarely as part of national/international cooperation projects. This diverges from the entomological research in the region, which holds eminent teaching and research centres and post-graduate courses on applied (e.g., medical and agricultural) entomology.

There is no published data from the actual experience of local forensic scientists in elucidating murder cases. The intricate police system in Brazil, aggravated by the insufficient number of professionals in the region, does not entitle scientific research as a priority for forensic experts, which explains the scarcity of criminal experts involved in ongoing cooperation projects with local universities. Most data on necrophagous species are limited to abstracts from local congresses and dissertations from undergraduate and post-graduate courses, which hampers the validation of the main findings. Publication in international journals is scant so far.

The restricted visibility and limited impact of data generated in the region may reflect not only the recent initiatives on this topic, but also limitations in the scope of the studies, such as: non-innovative design, lack of replication and statistical analysis, short-term field collection, among others. Legal, bureaucratic and ethical restraints limit the use of human cadavers in entomological surveys. The availability of bodies is highly irregular and procedures related to medicolegal practices (e.g., washing and disinfecting the cadavers) eliminate entomological evidence. Access to cadavers is limited to authorized personnel and authorization for external scientists requires a tremendous degree of bureaucracy. This fact compromises the reliability of data collected in broader inventories. To illustrate that, when dipteran species are analysed according to previous register on human cadavers, the diversity of dipterans is significantly reduced (Table V), and only a few had been actually used as valid evidence in criminal investigations, according to specialized literature.

Most critical of all is the superficial taxonomic identifi- 
cation, especially for Coleoptera and for dipteran families that need more complex examinations such as the morphological analysis of the male genitalia, as it is the case of Sarcophagidae. It is not impossible that inaccuracies in the identification may be manifested in the data presented here. The lack of experts on systematics hinders significant contributions that field surveys may attempt at, since the identification for most cases reported here is insufficient to establish the medico-legal importance of the species. Molecular tools devised to facilitate insect identification have not yet been employed in practical research in the region.

\section{CONCLUSIONS}

The establishment of forensic entomology as a reliable tool in the routine of forensic scientists in Northeastern Brazil faces challenges common to many developing countries exposed to high indices of violence. Yet, it could be significantly improved if standardized methods were addressed, based on sound knowledge on insects' systematics, bionomics and ecology. This methodological standardization should guarantee that entomological evidence is reliable and repeatable - especially when biological variations are inherent to such diversity of environments and resources.

Inventories of necrophagous species are not valuable unless data on other variables (environmental, to start with) that influence insect colonisation are also quantified. Furthermore, the lack of molecular and genetic studies is of special concern, since this type of information is essential to understand variations in development, colonization and survival - all of which influence PMI estimates (Tomberlin et al. 2011). Given the initial stage of the research on forensic entomology in Northeastern Brazil, it is expected that a substantial amount of time will elapse before entomological evidence is incorporated in the practice of the forensic investigator. The lack of cooperation between local entomologists, scientific police and lawyers is a major obstacle. Cases of entomological evidence playing a key role in legal processes have not been publicized in the nine states of the region - unlike increasing number of cases elsewhere in Brazil (Oliveira-Costa \& Mello-Patiu 2004; PujolLuz et al. 2006; Kosmann et al. 2011). Multidisciplinary studies involving aspects of bionomics, genetics, behaviour and molecular biology of necrophagous species start to be developed at local universities. Through this integration, it is expected that a more comprehensive overview of the actual diversity of necrophagous species in Northeastern Brazil, and their relevance for medico-legal investigations, will begin to emerge.

\section{ACKNOWLEDGEMENTS}

We thank all scientists who shared their data for this review, particularly Dr. Arlene Bezerra Santos (UFRPE) and Dr. Carla Lima Bicho (UEPB), CNPq (Ministério da Ciência e Tecnologia, Brazil) for the scholarship to the second author and Dr. Trev Williams (Inecol, Mexico) and Dr. Patricia Thyssen (UFPel, Brazil) for critical comments on the manuscript.

\section{REFERENCES}

Almeida, L. M. \& K. M. Mise. 2009. Diagnosis and key of the main families and species of South American Coleoptera of forensic importance. Revista Brasileira de Entomologia 53: 227-244.

Andrade, H. T. A.; A. A. Varela-Freire; M. J. Araujo Batista \& J. F. Medeiros. 2005. Calliphoridae (Diptera) coletados em cadáveres humanos no Rio Grande do Norte. Neotropical Entomology 34: 855-856.

Arnett Jr., R.H.; M. C. Thomas; P. E. Skelley \& J. H. Frank. 2002. American Beetles vol. 2: Polyphaga: Scarabaeoidea through Curculionoidea. Chicago, CRC Press. 880 p.

Barreto, M.; M. E. Burbano \& P. Barreto. 2002. Flies (Calliphoridae, Muscidae) and beetles (Silphidae) from human cadavers in Cali, Colombia. Memórias do Instituto Oswaldo Cruz 97: 137-138.

Benecke, M. 1998. Six forensic entomology cases: description and commentary. Journal of Forensic Sciences 43: 797-805.

Benecke, M. \& R. Lessig. 2001. Child neglect and forensic entomology. Forensic Science International 120: 155-159.

Byrd, J. H. \& J. L. Castner. 2010. Forensic Entomology: the utility of arthropods in legal investigations, $2^{\text {nd }} \mathrm{ed}$, Boca Raton, CRC Press, $705 \mathrm{p}$.

de Carvalho, C. J. B.; M. S. Couri; A. C. Pont; D. Pamplona \& S. M. Lopes. 2005. A catalogue of the Muscidae (Diptera) of the Neotropical Region. Zootaxa 860: 1-282.

de Carvalho, C. J. B. \& C. A. Mello-Patiu. 2008. Key to the adults of the most common forensic species of Diptera in South America. Revista Brasileira de Entomologia 52: 390-406.

Carvalho, L. M. L.; P. J. Thyssen; A. X. Linhares \& F. A. B. Palhares. 2000. A checklist of arthropods associated with pig carrion and human corpses in southeastern Brazil. Memórias do Instituto Oswaldo Cruz 95:135-138.

Carvalho, L. M. L.; P. J. Thyssen; M. L. Goff \& A. X. Linhares. 2004 Observations on the succession patterns of necrophagous insects on a pig carcass in a urban area of southeastern Brazil. Aggrawal's Internet Journal of Forensic Medicine and Toxicology 5: 33-39.

Catts, E. P. \& M. L. Goff. 1992. Forensic Entomology in criminal investigation. Annual Review of Entomology 37: 253-272.

Couri, M. S.; G. P. da S. Barros \& M. P. Orsini. 2008. Dipterofauna do Arquipélago de Fernando de Noronha (Pernambuco, Brasil). Revista Brasileira de Entomologia 52: 588-590.

Cruz, T. M. \& S. D. Vasconcelos. 2006. Entomofauna de solo associada à decomposição de carcaça de suíno em um fragmento de Mata Atlântica de Pernambuco, Brasil. Biociências 14: 193-201.

Cruz, T. M. 2008. Diversidade e sucessão ecológica de insetos associados à decomposição animal em um fragmento de Mata Atlântica de Pernambuco, com ênfase em Calliphoridae (Diptera). Dissertation, Recife, Universidade Federal Pernambuco, 110 p.

Disney, R. H. L. 2008. Natural history of the scuttle fly, Megaselia scalaris. Annual Review of Entomology 53: 39-60.

Endres, A. A.; M. I. M. Hernandez \& A. J. Creão-Duarte. 2005. Considerações sobre Coprophanaeus ensifer (Germar) (Coleoptera, Scarabaeidae) em um remanescente de Mata Atlântica no Estado da Paraíba, Brasil. Revista Brasileira de Entomologia 49: 427-429.

Ferreira, M. J. M. 1978. Sinantropia de Dípteros muscóideos de Curitiba, Paraná I: Calliphoridae. Revista Brasileira de Biologia 38: 445-454.

Grassberger, M.; E. Friedrich \& C. Reiter. 2003. The blowfly Chrysomya albiceps (Wiedemann) (Diptera: Calliphoridae) as a new forensic indicator in Central Europe. International Journal of Legal Medicine 117: 75-81.

Greenberg, B. \& J. D. Wells. 1998. Forensic use of Megaselia abdita and M. scalaris (Phoridae: Diptera): case studies, development rates, and eggs structure. Journal of Medical Entomology 35: 205-209.

Kosmann, C.; M. P. Macedo; T. A. F. Barbosa \& J. R. Pujol-Luz. 2011. Chrysomya albiceps (Wiedemann) and Hemilucilia segmentaria (Fabricius) (Diptera, Calliphoridae) used to estimate the postmortem interval in a forensic case in Minas Gerais, Brazil. Revista Brasileira de Entomologia 55: 621-623.

Kulshrestha, P. \& D. K. Satpathy. 2001. Use of beetles in forensic entomology. Forensic Science International 120: 15-17. 
Lord, W. D.; M. L. Goff; T. R. Adkins \& N. H. Haskell. 1994. The black soldier fly Hermetia illucens (Diptera: Stratiomyidae) as a potential measure of human postmortem interval: observations and case histories. Journal of Forensic Sciences 39: 215-222.

Mariluis, J. C.; J. A. Schnack; P. R. Mulieri \& J. P. Torretta. 2007. The Sarcophagidae (Diptera) of the coastline of Buenos Aires City, Argentina. Journal of the Kansas Entomological Society 80: 243-251.

Marquez-Luna, J. 2001. Especies necrofilas de Staphylinidae (Insecta: Coleoptera) del municipio de Tlayacapan, Morelos, Mexico. Folia Entomologica Mexicana 40: 93-131.

Mayer, A. C. G. 2009. Comunidade de artrópodes de solo com ênfase em Insecta (Coleoptera) nas circunvizinhanças de uma carcaça animal em um fragmento de Mata Atlântica de Pernambuco. Dissertation, Universidade Federal Pernambuco, Recife, 67 p.

Nilo, T. A. 2007. Análise do processo de decomposição e sucessão entomológica em carcaças de roedores em diferentes ambientes no município de Salvador, BA. Dissertation, Salvador, Universidade Federal Bahia, $89 \mathrm{p}$.

Oliveira T. P. B. 2009. Diversidade de Diptera de Importância Forense em Diferentes Biomas da Zona da Mata de Pernambuco. Dissertation, Universidade Federal de Pernambuco, 56 p.

Oliveira, T. C. \& S. D. Vasconcelos. 2010. Insects (Diptera) associated with cadavers at the Institute of Legal Medicine in Pernambuco, Brazil and its implications for forensic entomology. Forensic Science International 198: $97-102$.

Oliveira-Costa, J. \& C. A. Mello-Patiu. 2004. Application of forensic entomology to estimate the postmortem interval (PMI) in homicide investigations by Rio de Janeiro Police Department in Brazil. Aggrawal's Internet Journal of Forensic Medicine and Toxicology 5: 40-44.

Oliveira-Costa, J. 2011. Entomologia forense: quando os insetos são vestígios, $3^{\mathrm{a}}$ ed. Campinas, Millennium, $522 \mathrm{p}$

Pape, T. 1996. Catalogue of the Sarcophagidae of the World (Insecta: Diptera). Memoirs on Entomology, International, volume 8, 558 p.

Pujol-Luz, J. R.; H. Marques; A. Ururahy-Rodrigues; J. A. Rafael; F. H. A. Santana; L. C. Arantes \& R. Constantino. 2006. A forensic entomology case from the Amazon rain forest of Brazil. Journal of Forensic Sciences 51: 1151-1153.
Pujol-Luz, J. R.; L. C. Arantes \& R. Constantino. 2008. One hundred years of forensic entomology in Brazil (1908-2008). Revista Brasileira de Entomologia 52: 485-492.

Schroeder, H.; H. Klotzbach; L. Oesterhelweg \& P. Püschel. 2002. Larder beetles (Coleoptera, Dermestidae) as an accelerating factor for decomposition of a human corpse. Forensic Science International 127: $231-236$.

Shewell, G. E. 1987. Sarcophagidae, p. 1159-1186. In: McAlpine, J. F.; B. V. Peterson; G. E. Shewell; H. J. Teskey; J. R. Vockeroth \& D. M. Wood (eds.) Manual of Neartic Diptera, volume 2, Agriculture Canada Monograph 28.

Smith, K. G. V. 1986. A Manual of Forensic Entomology. London, Natural History Museum, $205 \mathrm{p}$

Sukontason, K.; P. Narongchai; C. Kanchai; K. Vichairat; P. Sribanditmongkol; T. Bhoopat; H. Kurahashi; M. Chockjamsai; S. Piangjai; N. Bunchu; S. Vongvivach; W. Samai; T. Chaiwong; R. Methanitikorn; R. Ngern-Klun; D. Sripakdee; W. Boonsriwong; S. Siriwattanarungsee; C. Srimuangwong; B. Hanterdsith; K. Chaiwan; C. Srisuwan; S. Upakut; K. Moopayak; R. C. Vogtsberger; J. K. Olson \& K. L. Sukontason. 2007. Forensic Entomology in Thailand: a review of cases from 2000 to 2006. Parasitology Research 101: 1417-1423.

Tomberlin, J. K.; R. Mohr; M. E. Benbow; A. M. Tarone \& S. VanLaerhoven. 2011. A roadmap for bridging basic and applied research in Forensic Entomology. Annual Review of Entomology 56: 401-421.

Velázquez, Y.; C. Magaña; A. Martinez-Sánchez \& S. Rojo. 2010. Diptera of forensic importance in the Iberian Peninsula: larval identification key. Medical and Veterinary Entomology 24: 293-308.

Waiselfisz, J. J. 2011. Mapa da Violência 2012 - Os Novos Padrões da Violência Homicida no Brasil. São Paulo, Instituto Sangari, 245 p.

Ward, D. F.; T. R. New \& A. L. Yen. 2001. Effects of pitfall trap spacing on the abundance, richness and composition of invertebrate catches. Journal of Insect Conservation 5: 47-53.

Wells, J. D. \& B. Greenberg. 1992. Interaction between Chrysomya rufifacies and Cochliomyia macellaria (Diptera: Calliphoridae): the possible consequences of an invasion. Bulletin of Entomological Research 82: 133-137.

Zumpt, F. 1965. Myasis in man and animals in the Old World. London. Butterworths, $267 \mathrm{p}$

Received 25/9/2011; accepted 13/2/2012

Editor: Reginaldo Constantino 